\section{Ingeniería}

CONTENIDO

Artículos

Análisis numérico de un sistema de aire acondicionado empleando
mallas estructuradas y no estructurades A

Funciones de regresión para caudales extremos en la Vertiente Rojas, Nazareth; Aguilar, José Francisco; Solís, Hernán. Evaluación de daños por agresión ambiental en viviendas
de concreto reforzado. Solís, Rómel; Moreno, Eric; Jiménez, Felipe; Rosas, Victorino.

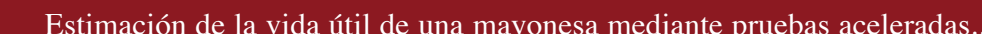
Estimación de la vida útil de una mayonesa
García, Claudia; Molina, Manuel.

Inestabilidad oscilatoria de tensión debido a los motores de inducción …….....................65-75 rde, Gustavo; Araya, Eddie.

Estabilidad y colapso de tensión en sistemas eléctricos Araya, Eddie.

Obtención de pulpa a la soda antraquinona de Tectona grandis creciendo en Costa Rica...........................

Protocolo evaluador para el manejo de desechos y la seguridad ocupacional
aplicado al laboratorio clínico del Hospital San Francisco de Asís de Grecia. Ruiz, Francisco.

9. Euler: su contex
Vargas, Celso.

10. Reserva de resistencia de edificios porticados de concreto armado disenaados conforme al ACI-318/ IBC 2006 ........ Análisis y comentarios

Herrera, Rodolfo.

breve semblanza.

Trabajos de Graduación 2007

Lista de proyectos de graduación de grado y posgrado

Normas

$\sum_{\text {EDTORAL }}$

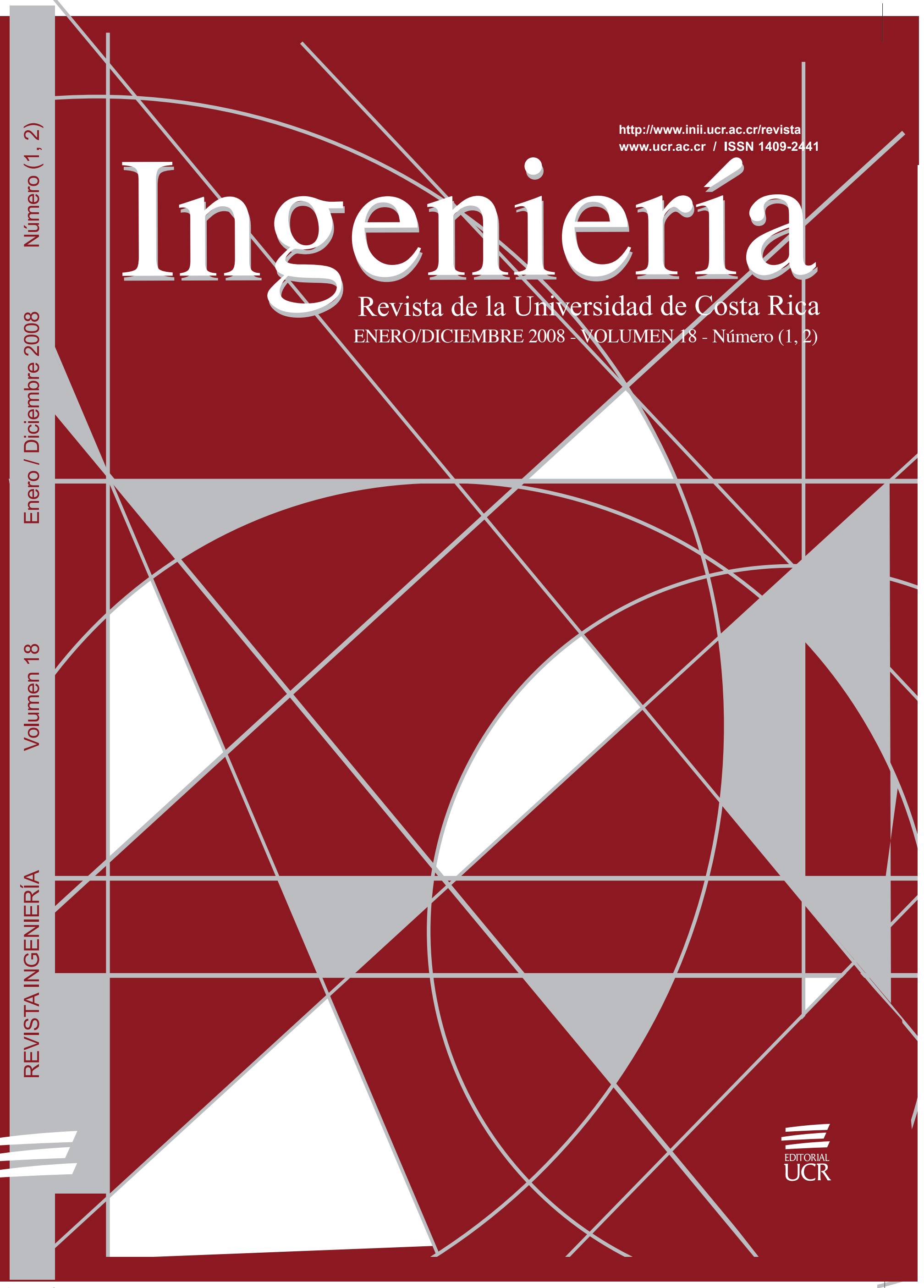




\title{
ANÁLISIS NUMÉRICO DE UN SISTEMA DE AIRE ACONDICIONADO EMPLEANDO MALLAS ESTRUCTURADAS Y NO ESTRUCTURADAS
}

\begin{abstract}
Resumen
En esta investigación se empleó el método de los volúmenes finitos para simular numéricamente el comportamiento térmico y fluidodinámico del aire en un sistema de distribución de aire acondicionado empleando mallas estructuradas y no estructuradas. Inicialmente se planteó el modelo matemático que rige el comportamiento del flujo de aire en el conducto de distribución y el sistema de ecuaciones obtenido fue cerrado mediante la aplicación del modelo de turbulencia k- $\varepsilon$. Se utilizaron dos técnicas para discretizar el dominio de cálculo: mallado estructurado y mallado no estructurado. Los resultados obtenidos en esta investigación indican que ambos métodos de discretización espacial resultan eficientes, desde el punto de vista numérico, en la simulación del comportamiento del aire en este tipo de sistemas.
\end{abstract}

Palabras clave: mallas estructuradas, mallas no estructuradas, volúmenes finitos, sistema de distribución de aire acondicionado.

\begin{abstract}
This article shows the result of using the finite volume method to numerically simulate the thermal and fluid behavior of an air conditioning distribution system, using both structured and non-structured meshing. Initially, the mathematical model that rules the airflow through ducts was posed and the resulting system of equations was solved applying the k- $\varepsilon$ turbulence model. Two techniques were used to discretize the calculus domain: structured and nonstructured meshing. The results indicate that both methods are efficient from a numerical point of view regarding the simulation of airflow in this kind of system.
\end{abstract}

Key words: structured meshes, non-structured meshes, finite volumes, air conditioning distribution systems.

Recibido: 25 de febrero del 2008 • Aprobado: 16 de septiembre del 2008

\section{INTRODUCCIÓN}

En un flujo térmico turbulento, la transferencia de calor está asociada a las características del movimiento caótico presente en el flujo, estando estrechamente relacionada con las fluctuaciones de las propiedades del flujo en espacio y tiempo y con los procesos de transporte físico de la turbulencia (Peng, 1998).

Debidoaquelasolución analítica es accesible sólo para pocas situaciones de flujo, las investigaciones están limitadas a los experimentos o a los métodos numéricos. Los experimentos usualmente predicen correctamente los efectos globales, mientras que los efectos locales de las variables son muy difíciles de medir. Los experimentos son también muy costosos y deben ser diseñados cuidadosamente para poder obtener resultados confiables. En contraste, las técnicas numéricas proveen información global y local de las variables en un corto período de tiempo reduciendo significativamente el costo de una investigación (Emvin, 2000).

En los últimos años, gracias a la rápida evolución de los computadores, ha surgido 
un área de la computación científica conocida como Dinámica de Fluidos Computacional (Computational Fluid Dynamics, CFD), mediante la cual es posible predecir el comportamiento de un fluido a través de métodos numéricos. La $C F D$ ha sido aplicada ampliamente en diversos problemas de flujo en ingeniería; un campo en el cual el uso de la $C F D$ está incrementándose activamente es en el diseño, mejoramiento y diagnóstico de sistemas de acondicionamiento de aire. Uno de los objetivos básicos de un sistema de aire acondicionado es el de controlar la temperatura y la calidad del aire en el ambiente, y ha sido demostrado que mediante el uso de técnicas $C F D$ es posible obtener una aproximación eficiente en cuanto a la transferencia de calor y a las condiciones del flujo de aire en un ambiente acondicionado (Peng, 1998).

Uno de los métodos comúnmente empleados en los códigos de la $C F D$ es el método de los volúmenes finitos (Finite Volume Method, $F V M$ ). Mediante la aplicación de este método es posible discretizar los problemas basándose en las leyes de conservación de la física, es decir, en dinámica de fluidos, transferencia de calor y masa. El FVM puede ser usado para discretizar dominios computacionales con geometrías complejas arrojando resultados confiables. Gracias a su propiedad de conservación local de los flujos, este esquema es atractivo para problemas donde el flujo debe ser preservado (Janka, 2001).

Es necesario hacer una elección acertada del método de discretización espacial a emplear en el dominio de cálculo del sistema simulado; para esto debe seleccionarse el tipo de mallado que mejor se ajuste a la geometría del dominio y a las características del flujo modelado.

Esta investigación se realiza con el propósito de encontrar el tipo de discretización espacial que resulte más eficiente en la simulación de sistemas de distribución de aire acondicionado. Para ello se realizan simulaciones numéricas del flujo en un sistema de distribución de aire acondicionado empleando el método de los volúmenes finitos mediante el paquete computacional $\mathrm{CFX}^{\mathrm{TM}}$; aplicando el modelo de turbulencia k-epsilon y empleando, para una misma geometría del conducto, dos tipos de discretización espacial: mallas estructuradas y mallas no estructuradas.
Como no se dispone de una solución real para este caso de estudio, las comparaciones entre los modelos simulados se establecerán con base en los residuos calculados para cada solución y al costo computacional de las mismas. El tipo de discretización espacial que mejor se adapte al caso estudiado será el que presente un mejor balance entre la exactitud de sus resultados y su costo computacional.

En años anteriores se han llevado a cabo investigaciones en el área de $C F D$ con el propósito de evaluar las condiciones de flujo del aire en sistemas de ventilación y aire acondicionado. Entre estas investigaciones se pueden citar las siguientes.

Cho y Kim (1998) realizaron una investigación sobre las características del flujo de aire y transferencia de calor en un sistema de aire acondicionado automotriz con el propósito de analizar algunos problemas de diseño. La distribución del flujo de aire y la temperatura del aire a la salida de los ductos, fueron analizadas empleandola Dinámica de Fluidos Computacional y el modelo $\mathrm{k}-\varepsilon$ estándar de turbulencia. Los resultados obtenidos coincidieron con la data experimental, demostrando que la Dinámica de Fluidos Computacional puede ser una herramienta muy útil en el diseño en sistemas de ventilación y aire acondicionado.

Kelso, Marshall y Baker (2001) citados por Pérez (2004), realizaron un estudio numérico del flujo de aire dentro de la caja mezcladora de una unidad de manejo de aire, empleando el código comercial CFX y el modelo de turbulencia $\mathrm{k}-\varepsilon$ estándar. Este estudio fue realizado con el objeto de evaluar la eficiencia de mezclado de dos corrientes de aire con diferentes temperaturas y se obtuvieron resultados numéricos satisfactorios.

Schneider y Maliska (2002), emplearon un método numérico para la solución de flujos incompresiblesbidimensionalesutilizandoelmétodo de volúmenes finitos con mallas no estructuradas. Estos investigadores aplicaron la solución simultánea de las ecuaciones de conservación de masa y energía obteniendo una matriz de coeficientes única. La formulación aplicada, al ser comparada con los métodos segregados, redujo la complejidad del algoritmo. Schneider y Maliska 
(2002) encontraron que las mallas no estructuradas se adaptan mejor tanto a los dominios simples como a los complejos, permitiendo el refinamiento local en regiones específicas.

\section{METODOLOGÍA}

\subsection{Modelo físico}

El modelo físico consiste en un sistema de distribución de aire acondicionado, diseñado mediante la aplicación del método de fricción constante, que recibe $0,57 \mathrm{~m}^{3} / \mathrm{s}$ de aire frío del equipo acondicionador y lo suministra al espacio mediante tres salidas de $0,2 \mathrm{~m}$ por $0,2 \mathrm{~m}$. Las características geométricas y dimensionales del sistema se muestran en las Figuras 1 y 2 .

\subsection{Modelo matemático}

El modelo matemático de este sistema de distribución está conformado por la ecuación de continuidad, las ecuaciones de movimiento y la ecuación de conservación de la energía, aplicadas a un flujo tridimensional, estacionario y no isotérmico de un fluido viscoso con propiedades físicas constantes. Este modelo matemático emplea varias simplificaciones que facilitan su solución, pero sin alterar su validez, estas simplificaciones se presentan a continuación:

a) Flujo incompresible.

b) Fluido viscoso newtoniano.

c) Fluido con propiedades físicas constantes: $\rho$, $\mu$ y K.

d) Estado estacionario.

Es importante destacar que en el comportamiento de un sistema de distribución de aire acondicionado existe una condición transitoria originada en el arranque del sistema o por la variación de las temperaturas en el interior del espacio, debido al cambio de las condiciones de la atmósfera exterior por hora del día. A pesar de esto, el modelo matemático a aplicar en esta investigación empleará la idealización de estado estacionario con el objeto de simplificar las ecuaciones que rigen el comportamiento del sistema.

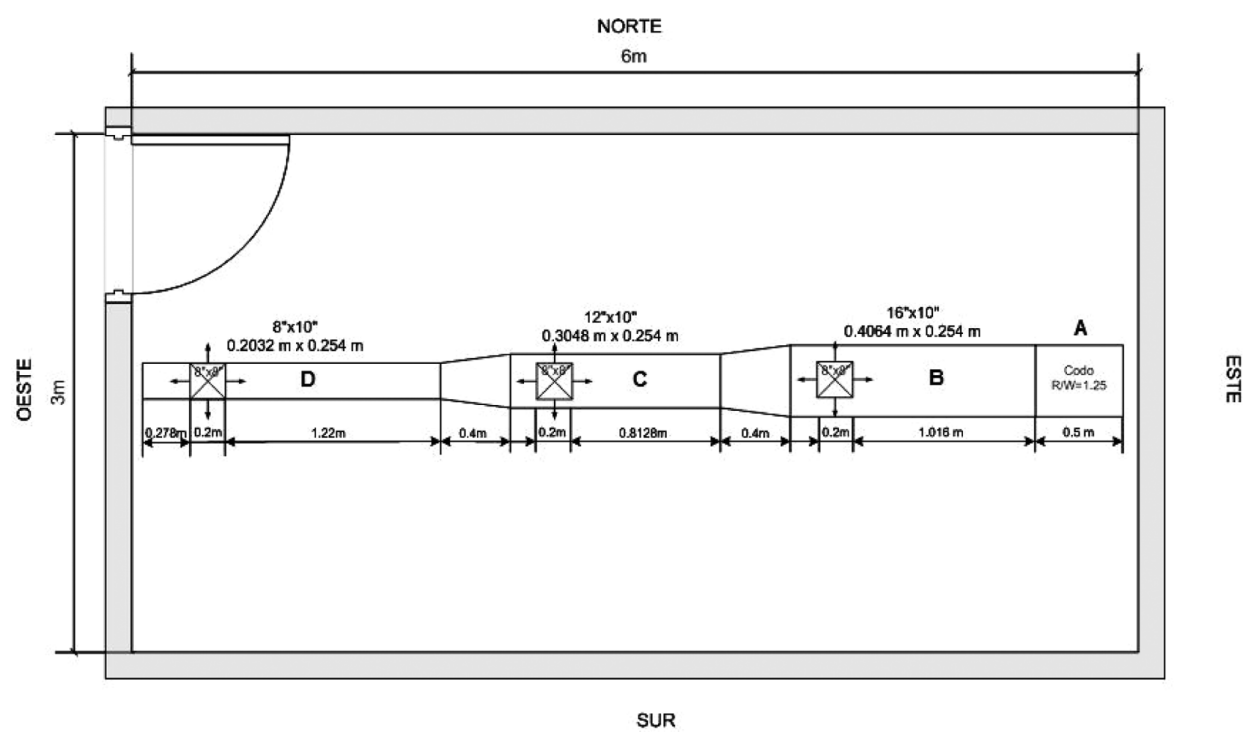

Figura 1. Dimensiones del sistema de distribución. Fuente: (Rodríguez \& Verde, 2005). 


\subsubsection{Ecuaciones de conservación}

Como resultado de las idealizaciones citadas anteriormente, la ecuación de continuidad y de la aplicación del concepto de la media temporal de Reynolds, las ecuaciones de movimiento y la ecuación de la energía, expresadas en coordenadas rectangulares, quedan simplificadas de la siguiente manera:

Ecuación de continuidad de tiempo ajustado en su forma vectorial

$(\nabla \cdot \bar{v})=0$

Ecuación de movimiento de tiempo ajustado en su forma vectorial

$\rho \nabla \cdot(\bar{v} \otimes \bar{v})=-\nabla \bar{p}-\left[\nabla \cdot \bar{\tau}^{(l)}\right]-\left[\nabla \cdot \bar{\tau}^{(t)}\right]+\rho g_{z}$

Donde:

$\overline{\boldsymbol{\tau}}_{x x}{ }^{(t)}=\rho \overline{v_{x}{ }^{\prime} v_{x}{ }^{\prime}} ; \bar{\tau}_{x y}{ }^{(t)}=\rho \overline{v_{x}{ }^{\prime} v_{y}{ }^{\prime}} ;$ etc.

$\bar{\tau}_{x x}{ }^{(l)}=-\mu\left[2 \frac{\partial v_{x}}{\partial x}\right] ; \bar{\tau}_{y y}{ }^{(l)}=-\mu\left[2 \frac{\partial v_{y}}{\partial y}\right] ;$ etc.

Ecuación de la energía de tiempo ajustado en forma vectorial

Donde:

\subsubsection{Modelo de turbulencia $k-\varepsilon$}

Uno de los modelos de turbulencia más prominentes, el k- $\varepsilon$, ha sido implementado en la mayoría de los códigos de dinámica de fluidos computacional y es considerado el modelo estándar de la industria. Este modelo ha sido extensamente validado y ha demostrado ser estable y numéricamente robusto. En el software $\mathrm{CFX}^{\mathrm{TM}} \mathrm{el}$ modelo de turbulencia $k-\varepsilon$ utiliza la aproximación de la función de pared para mejorar la robustez y precisión de los resultados cuando el mallado en la región cercana a la pared es muy fino.

La energía cinética turbulenta, $\mathrm{k}$, está definida como la variación de las fluctuaciones en la velocidad y tiene las dimensiones de $\left(\mathrm{m}^{2} / \mathrm{s}^{2}\right), \varepsilon$ es la disipación de remolino turbulenta (la tasa a la cual las fluctuaciones de velocidad se disipan) y tiene las dimensiones de $\left(\mathrm{m}^{2} / \mathrm{s}^{3}\right)$.

El modelo k- $\varepsilon$ está basado en el concepto de la viscosidad remolino, y asume que la viscosidad turbulenta está ligada a la energía cinética turbulenta y la disipación mediante la relación (4).

$\mu_{t}=C_{\mu} \rho\left(\frac{k^{2}}{\varepsilon}\right)$

Donde:

$\mathrm{C}_{\mu}$ es una constante.

$$
\begin{aligned}
& \rho \hat{C} v \nabla \cdot(\bar{T} \bar{v})=-\left[\nabla \cdot \bar{q}^{(l)}\right]-\left[\nabla \cdot \bar{q}^{(t)}\right]+\mu \bar{\Phi}_{v}{ }^{(l)}+\mu \bar{\Phi}_{v}{ }^{(t)} \\
& \overline{\mathrm{q}}^{(1)}=-\mathrm{K} \nabla^{2} \overline{\mathrm{T}} \\
& \bar{q}_{x}^{(t)}=\rho \hat{C}_{V} \overline{v_{x}{ }^{\prime} T^{\prime}} ; \bar{q}_{y}{ }^{(t)}=\rho \hat{C}_{V}{\overline{v_{y}{ }^{\prime} T^{\prime}}}^{\prime} \bar{q}_{z}{ }^{(t)}=\rho \hat{C}_{V} \overline{v_{z}{ }^{\prime} T^{\prime}} \\
& \bar{\Phi}_{v}^{(l)}=2\left\{\left(\frac{\partial \bar{v}_{x}}{\partial x}\right)^{2}+\left(\frac{\partial \bar{v}_{y}}{\partial y}\right)^{2}+\left(\frac{\partial \bar{v}_{z}}{\partial z}\right)^{2}\right\}+\left\{\left(\frac{\partial \bar{v}_{x}}{\partial y}+\frac{\partial \bar{v}_{y}}{\partial x}\right)^{2}+\left(\frac{\partial \bar{v}_{x}}{\partial z}+\frac{\partial \bar{v}_{z}}{\partial x}\right)^{2}+\left(\frac{\partial \bar{v}_{y}}{\partial z}+\frac{\partial \bar{v}_{z}}{\partial y}\right)^{2}\right\} \\
& \overline{\boldsymbol{\Phi}}_{v}{ }^{(t)}=\sum_{i=1}^{3} \sum_{j=1}^{3}\left(\overline{\frac{\partial v_{i}{ }^{\prime}}{\partial x_{j}} \frac{\partial v_{i}{ }^{\prime}}{\partial x_{j}}}+\overline{\frac{\partial v_{i}{ }^{\prime}}{\partial x_{j}} \frac{\partial v_{j}{ }^{\prime}}{\partial x_{i}}}\right)
\end{aligned}
$$


Los valores de $\mathrm{k}$ y $\varepsilon$ vienen directamente de las ecuaciones diferenciales de transporte de la energía cinética turbulenta y de la tasa de disipación turbulenta.

$$
\begin{aligned}
& \rho \nabla \cdot(\bar{v} k)=\nabla \cdot\left[\left(\mu+\frac{\mu_{t}}{\sigma_{k}}\right) \nabla k\right]+P_{k}-\rho \varepsilon \\
& \rho \nabla \bullet(\bar{v} \varepsilon)=\nabla \bullet\left[\left(\mu+\frac{\mu_{t}}{\sigma_{\varepsilon}}\right) \nabla \varepsilon\right]+\frac{\varepsilon}{k}\left(C_{\varepsilon 1} P_{k}-C_{\varepsilon 2} \rho \varepsilon\right)
\end{aligned}
$$

Donde:

$\mathrm{C}_{\varepsilon 1}, \mathrm{C}_{\varepsilon 2}, \sigma_{k}$ y $\sigma_{\varepsilon}$ son constantes

$\mathrm{P}_{k}$ producción turbulenta debido a las fuerzas viscosas y a la fuerzas de flotación

$\mathrm{P}_{k}$ es modelada empleando la ecuación (7).

$$
P_{k}=\mu_{t} \nabla \bar{v} \cdot(\nabla \bar{v}+\nabla \bar{v})+P_{k b}
$$

Empleando el modelo de flotación de Boussinesq, $\mathrm{P}_{k b}$ es el término de producción de la flotación y está definido de la ecuación (8).

$$
P_{k b}=\frac{\mu_{t}}{\operatorname{Pr}_{t}} \rho \beta g \bullet \nabla T
$$

\subsection{Condiciones de contorno}

Una vez planteadas todas las ecuaciones de gobierno que rigen el comportamiento del flujo de aire en el sistema de distribución, es necesario definir las condiciones de contorno que permitirán dar solución a esas ecuaciones de conservación. Las características geométricas del dominio en estudio permitieron hacer las simulaciones considerando sólo la mitad de la geometría.

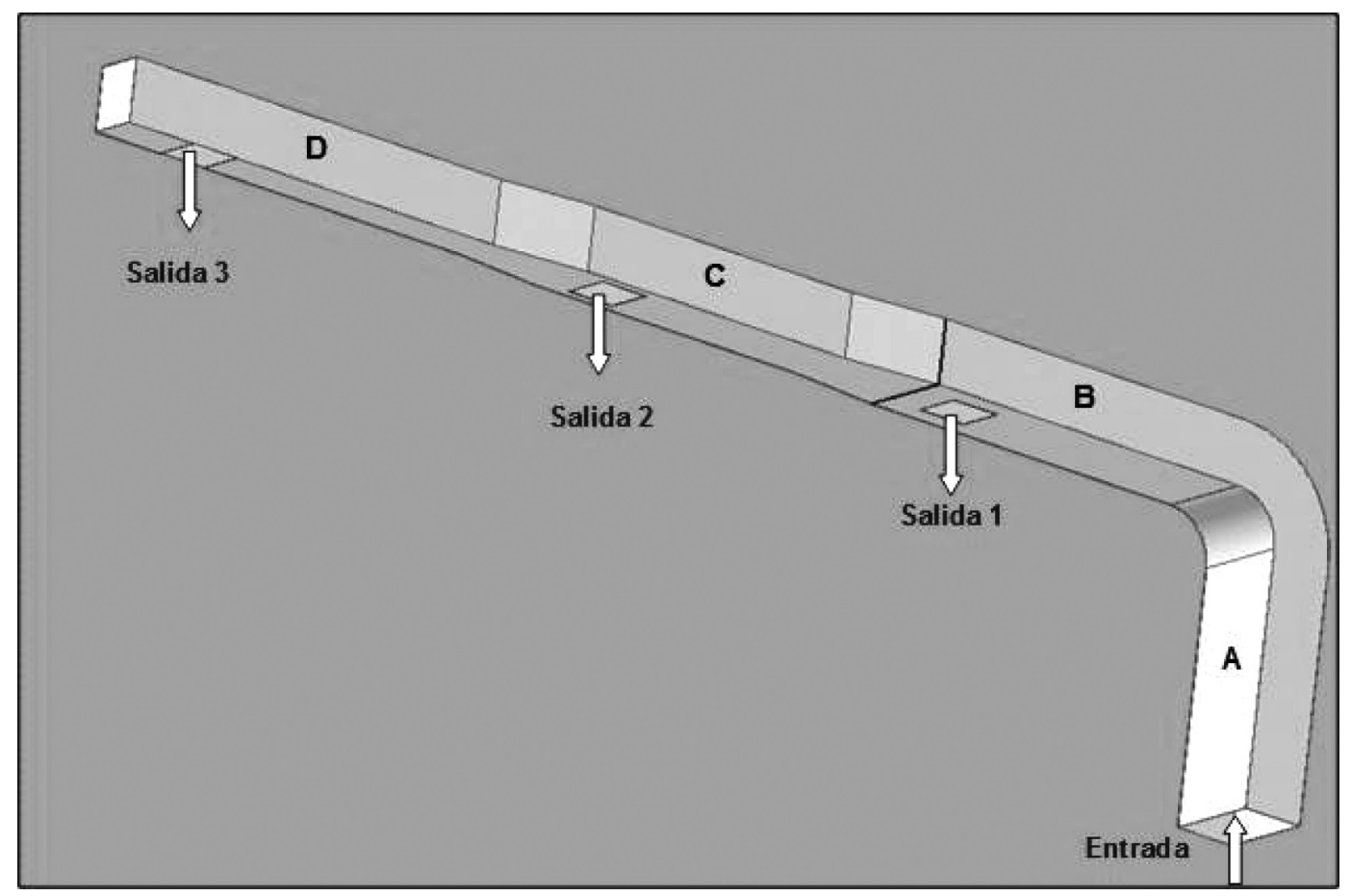

Figura 2. Vista isométrica del sistema de distribución.

Fuente: (Rodríguez \& Verde, 2005). 
Esto es una simplificación que se hace con el propósito de disminuir el costo computacional de la simulación aunque se sabe que es posible que esta aproximación introduzca cierto porcentaje de error en los resultados de la simulación. Las condiciones de borde del problema fueron impuestas en el dominio de la siguiente manera:

- Entrada:

$$
\begin{array}{rlrl}
\mathrm{v}_{x} & =0 & \mathrm{p}=\mathrm{p}_{e} \\
\mathrm{v}_{y}=0 & \mathrm{k}=\mathrm{k}_{e} \\
\mathrm{v}_{z}=\mathrm{v}_{e} & \varepsilon=\varepsilon_{e} \\
\mathrm{~T}=\mathrm{T}_{e} & &
\end{array}
$$

Donde:

$e$ son los valores de entrada de las variables.

- Salidas:

$\mathrm{p}=\mathrm{p}_{s}$

$\mathrm{k}=\mathrm{k}_{s}$

$\varepsilon=\varepsilon_{s}$

Donde:

$s$ son los valores de salida de las variables.

- Paredes:

Condición de no deslizamiento:

$\mathrm{V}_{\text {fluido }}=\mathrm{V}_{\text {pared }}=0$

Coeficiente de transferencia de calor:

$\mathrm{q}_{\mathrm{w}}=\mathrm{U}\left(\mathrm{T}_{\mathrm{b}}-\mathrm{T}_{\mathrm{nw}}\right)$

Donde:

U coeficiente global de transferencia de calor especificado

$\mathrm{T}_{b}$ temperatura de contorno especificada

$\mathrm{T}_{n w}$ temperatura en el nodo próximo a la pared.

\subsection{Solución numérica}

Las ecuaciones diferenciales con sus respectivas condiciones de borde descritas en el modelo matemático fueron resueltas a través del código comercial de Dinámica de Fluidos Computacional $\mathrm{CFX}^{\mathrm{TM}}$, el cual basa la solución en el método de los volúmenes finitos para mallas no estructuradas.
Para resolver las ecuaciones gobernantes del dominio en estudio se emplearon dos tipos de discretización espacial: mallado estructurado, el cual se caracteriza porque cada una de sus celdas o volúmenes internos tiene el mismo número de vecinos; y mallado no estructurado, donde cada volumen interno puede tener un número cualquiera de vecinos.

\subsubsection{Construcción del mallado estructurado}

Para la construcción de este mallado se empleó la técnica "Isomesh" la cual genera elementos en forma de hexaedros. La malla fue construida de forma manual con refinamiento en las zonas cercanas a las paredes del dominio, a las salidas de aire y en el tramo final del ducto, por ser estas las zonas en donde se requiere conocer con mayor precisión los valores de las distintas variables del flujo.

En la Figura 3 se muestra un corte transversal de la geometría mallada; en ella se observa cómo el tamaño de los elementos va disminuyendo gradualmente al aproximarse a las paredes del dominio. En las Figuras 4 y 5 se muestra un corte longitudinal de la geometría mallada, evidenciándose el refinamiento de malla en las salidas de aire y en la zona final del ducto.

\subsubsection{Construcción del mallado no estructurado}

Para la construcción del mallado no estructurado se empleó el método de Avance Frontal e Inflación (Advancing Front and Inflation, AFI), el cual produce una malla de superficie triangular empleando el método de Delaunay y una malla de volumen, mediante el método de Avance Frontal, que puede contener elementos en forma de tetraedros, prismas y pirámides.

En el dominio en estudio se construyó una malla no estructurada con refinamientos locales en las zonas próximas a las salidas de aire, se empleó el mecanismo de inflación en todas las paredes de la geometría y además se construyó una malla más refinada en la zona final del 


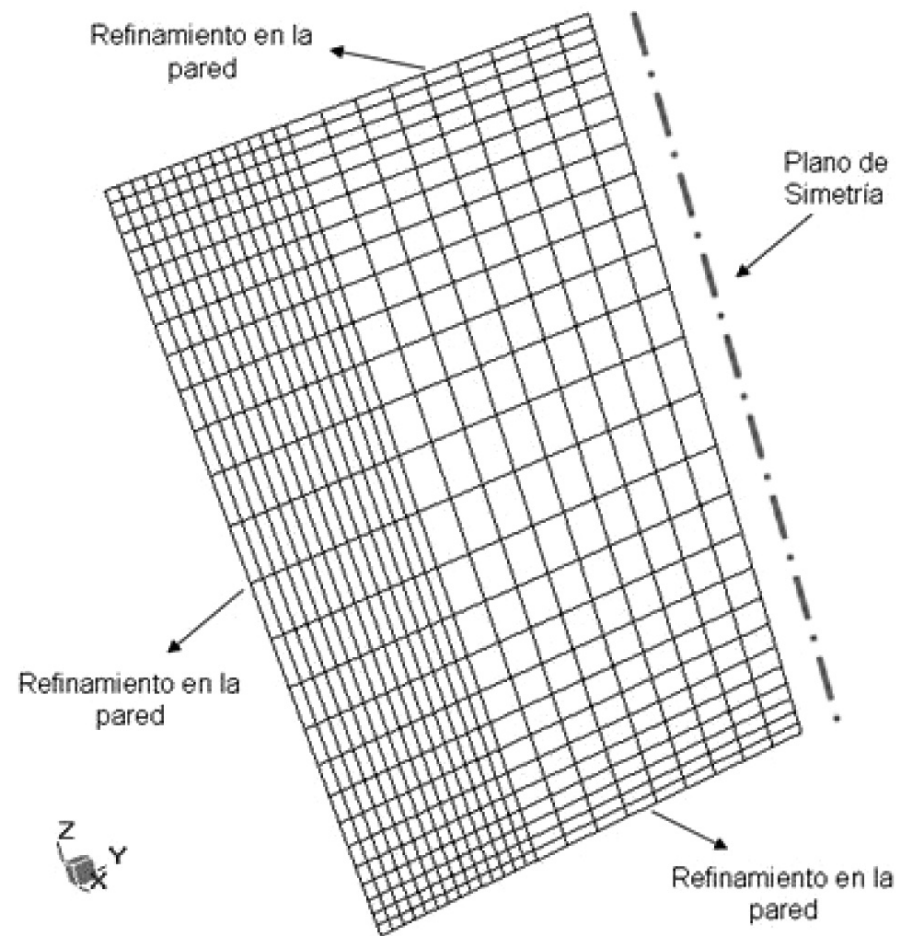

Figura 3. Refinamiento de la malla en las zonas cercanas a las paredes del dominio. Fuente: (Rodríguez \& Verde, 2005).

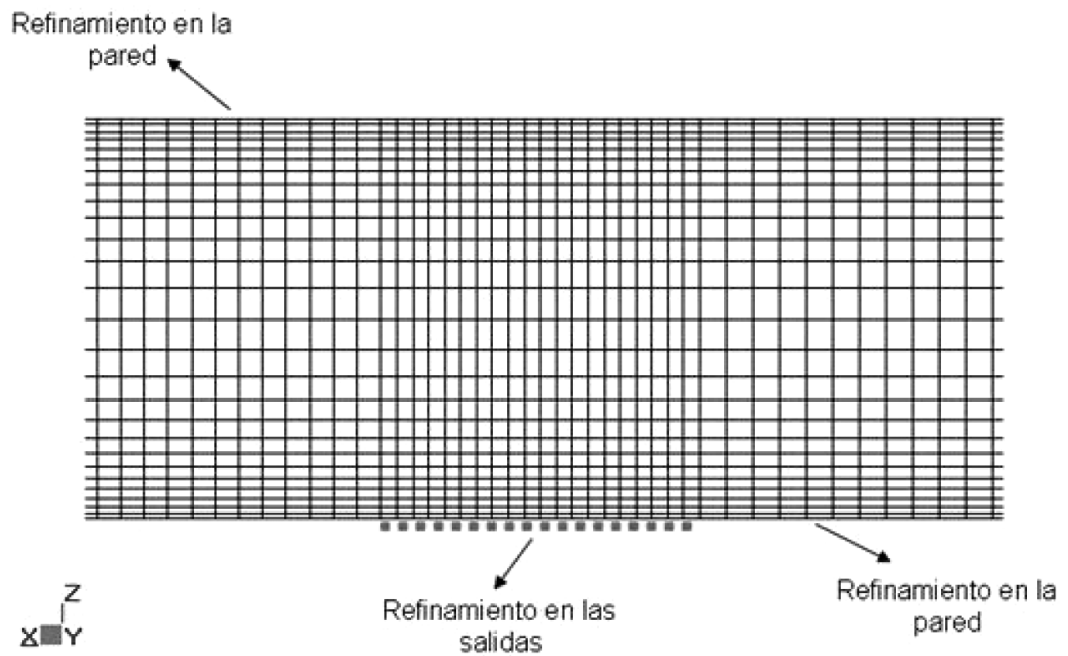

Figura 4. Refinamiento de la malla en las salidas de aire. Fuente: (Rodríguez \& Verde, 2005). 
ducto para observar con mayor exactitud el fenómeno de convección libre existente en esa región del dominio. En la Figura 6 se muestra un corte transversal y longitudinal de la malla construida.

Adicionalmente se generó un refinamiento de la malla en las zonas cercanas a las salidas de aire, este efecto fue logrado gracias al uso de un controlador de malla ubicado en la línea central de las salidas de aire. En la Figura 7 se muestra el refinamiento de la malla en la zona final del conducto.

\subsection{Parámetros de solución}

Para cada caso simulado se emplearon los parámetros de solución adecuados con el objeto de obtener, en la medida de lo posible, una buena tasa de convergencia. A continuación se explican, de forma general, las características de cada parámetro de solución.

\subsubsection{Acoplamiento presión-velocidad}

El software $\mathrm{CFX}^{\mathrm{TM}}$ utiliza un "solver" acoplado, el cual resuelve las ecuaciones hidrodinámicas (para la presión y la velocidad) como un sistema individual. Esta técnica de solución utiliza una discretización implícita completa de las ecuaciones en cualquier paso de tiempo (ANSYS, 2002).

Este software utiliza una malla colocada "unstaggered" para resolver el acoplamiento presión velocidad mediante la adición de un término adicional a la ecuación de conservación de la masa.

\subsubsection{Técnica multimalla algebraica (AMG):}

El proceso de esta técnica comprende la solución de las ecuaciones de conservación para una malla fina en las primeras iteraciones y luego en mallas “virtuales" progresivamente más gruesas. Los resultados obtenidos para la malla más gruesa son entonces interpolados hacia la malla fina original (ANSYS, 2002).

\subsubsection{Esquema de advección}

Esquema de Alta Resolución: en este esquema el factor de mezcla varía a través del dominio basado en el campo de solución local. En regiones de flujo con gradientes de variables bajos, el factor de mezcla será cercano a uno. En áreas donde los gradientes cambien bruscamente, el factor de mezcla será cercano a cero. Este esquema fue empleado en las simulaciones con el modelo k- $\varepsilon$ con los dos tipos de discretización espacial y para los dos materiales.

\subsubsection{Inicialización}

En los dos casos simulados en este trabajo fueron empleados valores iniciales automáticos para las diferentes variables del flujo.

\subsubsection{Criterio de convergencia}

El $\mathrm{CFX}^{\mathrm{TM}}$ calcula residuos normalizados para juzgar la convergencia de los problemas simulados. El residuo normalizado es empleado por el "solver" para detener automáticamente una corrida cuando ha sido alcanzado un nivel de residual especificado por el usuario. El software permite seleccionar el criterio de parada de la solución entre dos normas disponibles para el cálculo de los residuos.

La norma Raíz del Promedio de los Cuadrados (Root Mean Square, RMS), eleva al cuadrado el residual normalizado en cada volumen de control del dominio, luego suma esos cuadrados, los promedia y finalmente, saca la raíz cuadrada a este valor:

$$
R M S=\sqrt{\frac{1}{n} \sum_{i=1}^{n}(r)^{2}}
$$

\section{Donde:}

n número de volúmenes de control en el dominio

$r$ residual en cada uno de esos volúmenes 


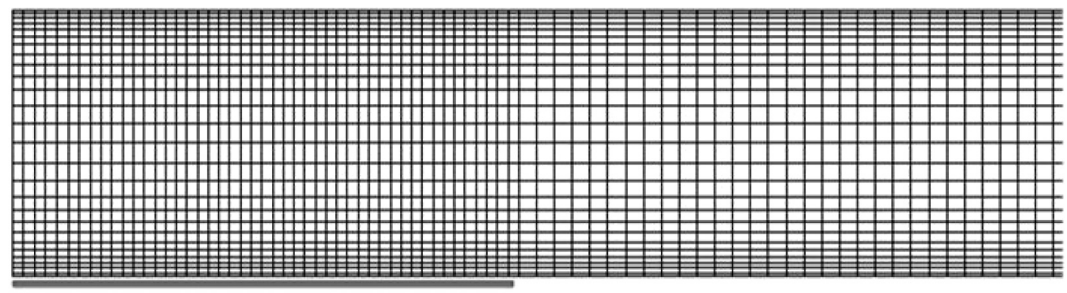

Refinamiento en el tramo

final de ducto

Figura 5. Refinamiento de la malla en el tramo final del ducto.

Fuente: (Rodríguez \& Verde, 2005).
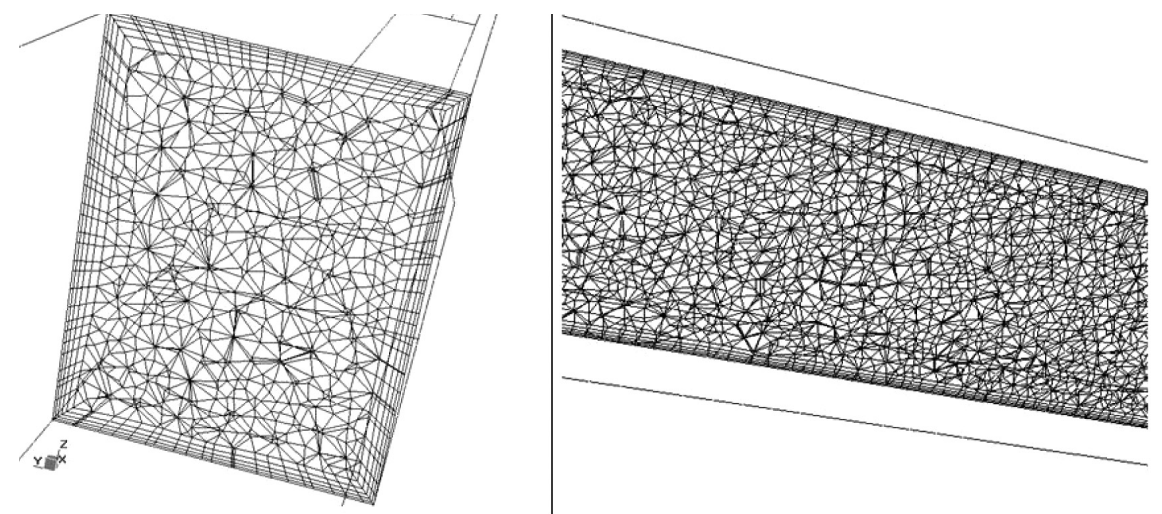

Figura 6. Corte transversal (derecho) y longitudinal (izquierdo) de la malla no estructurada. Fuente: (Rodríguez \& Verde, 2005).

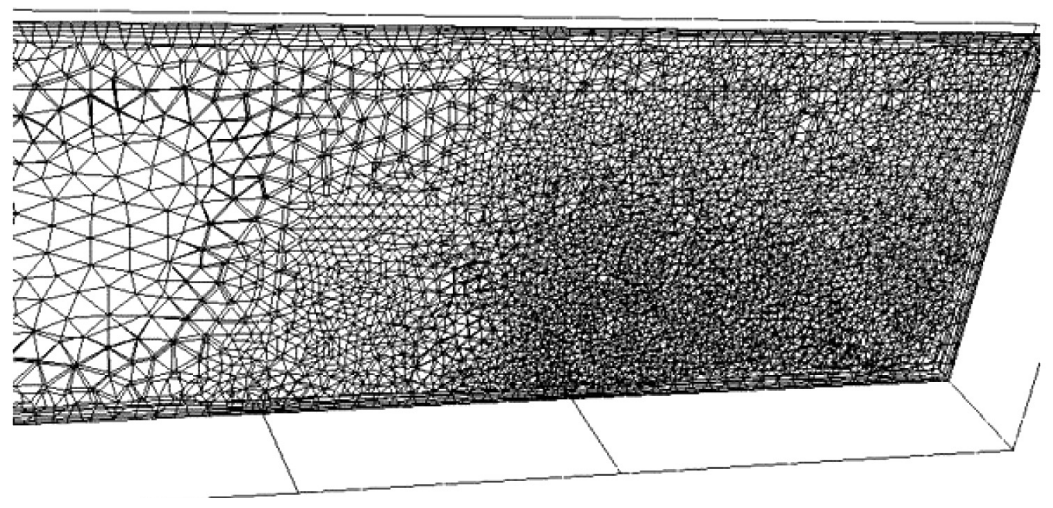

Figura 7. Refinamiento de la malla en la zona final del ducto. Fuente: (Rodríguez \& Verde, 2005). 
La segunda norma conocida como MAX establece que el valor del residual es igual al valor absoluto del máximo residuo en todo el dominio:

$M A X=\max |r|$

Para juzgar la convergencia del problema en estudio se seleccionó el límite de residual como $1 \cdot 10^{-5}$ en norma RMS.

\section{RESULTADOS}

\subsection{Simulación con mallado no estructurado}

Esta simulación requirió doscientas sesenta y una iteraciones con un tiempo de cálculo de $8 \mathrm{~h}$ con 16 min para alcanzar los niveles de convergencia requeridos, obteniéndose un rango de variación de temperatura en el dominio de 3,3648 K.

La Figura 8 muestra las curvas de residual en norma RMS para las velocidades y la masa obtenida en este caso simulado. Esta figura muestra que la Velocidad $U$ es la variable que requirió mayor número de iteraciones para alcanzar el nivel de convergencia especificado; esto se debe a que es la componente del vector velocidad que sufre mayores fluctuaciones a lo largo del dominio, por ser la velocidad principal del flujo. Adicionalmente, se observa que la curva de residual de masa es la que alcanza el nivel de convergencia requerido en menor número de iteraciones y su residuo final es del orden de $3,3 \cdot 10^{-7}$ RMS, mucho menor que el residuo final del resto de las variables; comportamiento que indica que la acumulación de masa en el dominio es bastante pequeña.

Se observa que en las curvas de las velocidades $\mathrm{U}$ y $\mathrm{W}$ se presentan oscilaciones repetitivas que dificultan la convergencia de la solución; esto puede deberse a distorsiones en la malla o a errores provenientes de la discretización de los términos convectivos en las ecuaciones de gobierno.

La curva de residual en Norma RMS para la entalpía se muestra en la Figura 9. En esta figura es posible notar que la entalpía alcanza el nivel de convergencia requerido mucho antes que las velocidades, en la iteración setenta aproximadamente, y su residuo final es también más pequeño.

Las curvas de residual en Norma RMS para la energía cinética turbulenta $\mathrm{k}$ y su tasa de disipación $\varepsilon$ se muestran en la Figura 10. El software CFX ${ }^{\mathrm{TM}}$ no toma en cuenta estas variables al momento de aplicar el criterio de convergencia especificado por el usuario; a pesar de esto se observa que tanto $\mathrm{k}$ como $\varepsilon$ alcanzan el límite de residual de $1 \cdot 10^{-5} \mathrm{RMS}$.

\subsection{Simulación con mallado estructurado}

Esta simulación requirió de ciento veinticuatro iteraciones para alcanzar el límite de convergencia de $1 \cdot 10^{-5} \mathrm{RMS}$, con un tiempo de cálculo de $7 \mathrm{~h}$ con $30 \mathrm{~min}$, obteniéndose un rango de variación de temperatura en el dominio de 9,799 K.

En la Figura 11 se muestran las curvas de residual en norma RMS para la velocidad en sus tres componentes y la masa. A diferencia del caso anterior, estas presentan un comportamiento suave, sin oscilaciones.

La curva de residual de la entalpía se muestra en la Figura 12. Esta curva también presenta un comportamiento regular, prácticamente sin oscilaciones, sin embargo, el valor del residuo final de la entalpía es mayor que el obtenido en el caso anterior, lo que genera una variación de temperatura en el dominio mucho mayor. Este comportamiento puede atribuirse a que, al no existir oscilaciones en las curvas de residual de velocidad, el límite de convergencia de la solución se alcanza mucho más rápido y esto no permite que el residual de entalpía disminuya tanto como en el caso anterior. Con el propósito de aclarar esta situación, se permitió que la solución avanzara para observar el comportamiento de la variable entalpía; encontrándose que, por debajo del valor de residual de $1 \cdot 10^{-5}$, la tasa de convergencia de esa variable era muy baja y por ende, no se justificaba continuar el proceso de cálculo.

En la Figura 13 se muestra la curva de residual de la energía cinética turbulenta $\mathrm{k}$ y su tasa de disipación $\varepsilon$. En estas curvas se observa que los residuos finales obtenidos para estas dos variables son mayores a los del modelo k- $\varepsilon$ en mallado no estructurado, debido también a la razón expuesta para el caso de la entalpía en el párrafo anterior. 


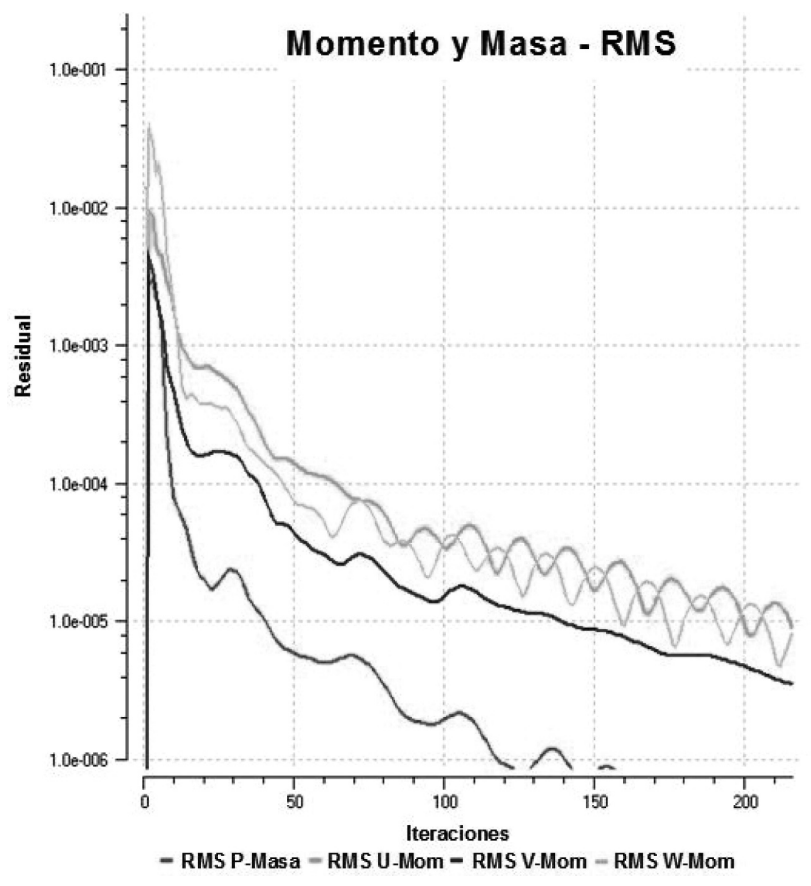

Figura 8. Curvas de residual en norma RMS para las ecuaciones de masa y momento en la simulación con malla no estructurada y modelo de turbulencia $\mathrm{k}-\varepsilon$.

Fuente: (Rodríguez \& Verde, 2005).

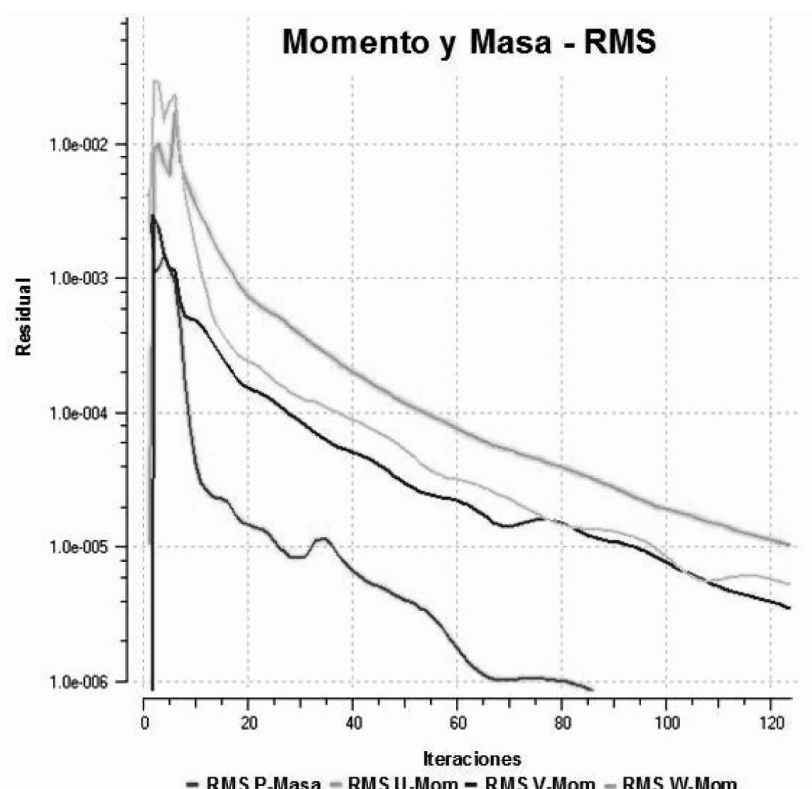

Figura 9. Curva de residual en norma RMS para la ecuación de la energía en la simulación con malla no estructurada y modelo de turbulencia k- $\varepsilon$.

Fuente: (Rodríguez \& Verde, 2005). 


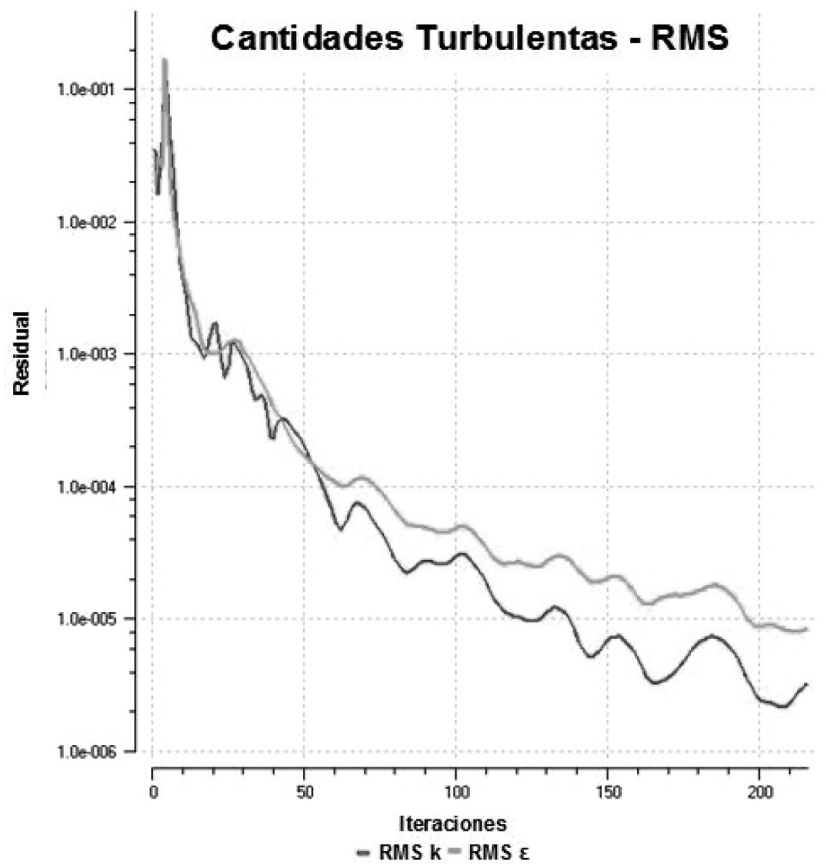

Figura 10. Curva de residual en norma RMS para la energía cinética turbulenta y su tasa de disipación en la simulación con malla no estructurada y modelo de turbulencia k- $\varepsilon$.

Fuente: (Rodríguez \& Verde, 2005).

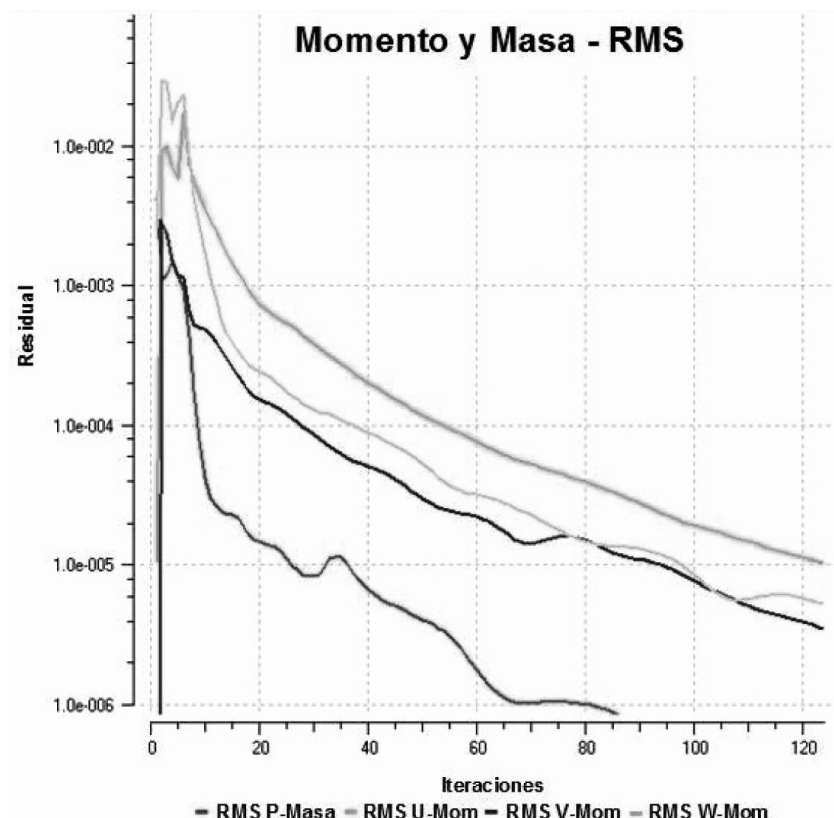

Figura 11. Curvas de residual en norma RMS para las ecuaciones de masa y momento en la simulación con malla estructurada y modelo de turbulencia k- $\varepsilon$.

Fuente: (Rodríguez \& Verde, 2005). 


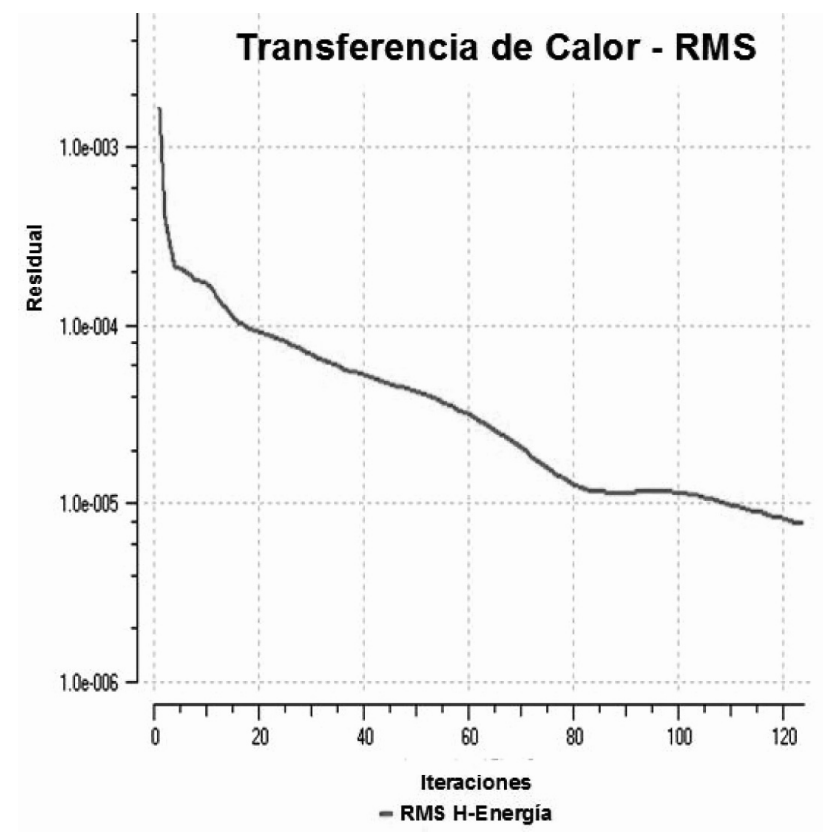

Figura 12. Curvas de residual en norma RMS para la ecuación de la energía en la simulación con malla estructurada y modelo de turbulencia $\mathrm{k}-\varepsilon$.

Fuente: (Rodríguez \& Verde, 2005).

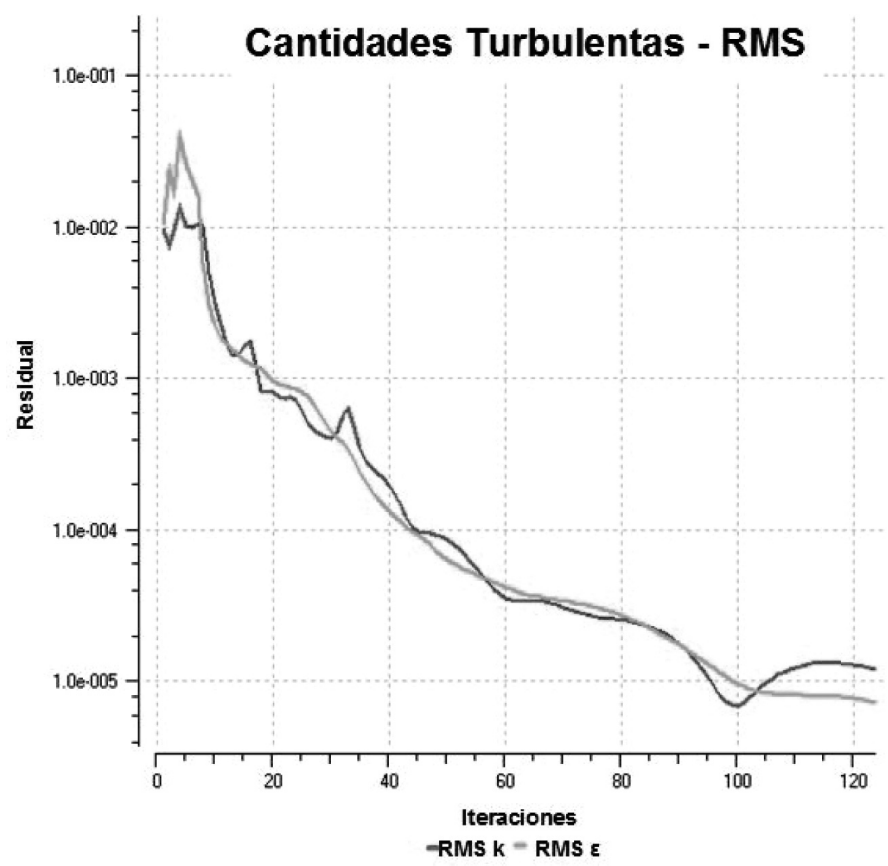

Figura 13. Curvas de residual en norma RMS para la energía cinética turbulenta y su tasa de disipación en la simulación con malla estructurada y modelo de turbulencia k- $\varepsilon$.

Fuente: (Rodríguez \& Verde, 2005). 


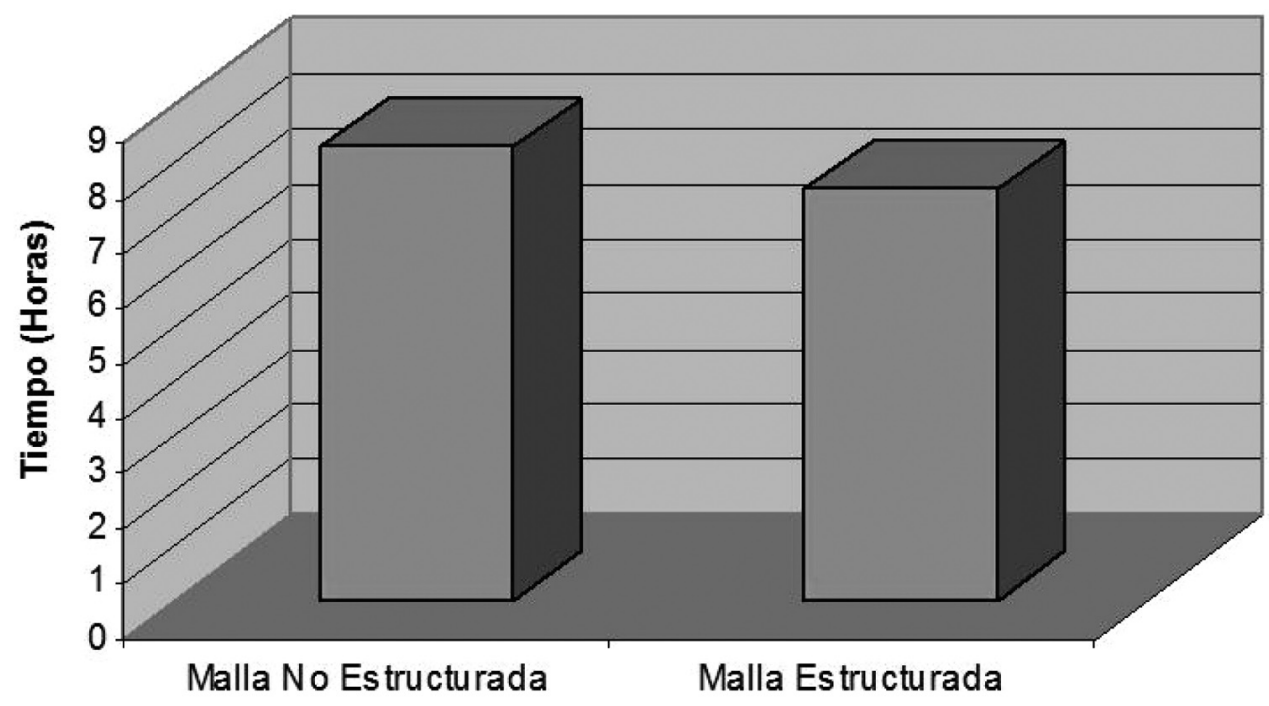

Figura 14. Comparación entre los tiempos de cálculo para los dos casos simulados. Fuente: (Rodríguez, 2007).

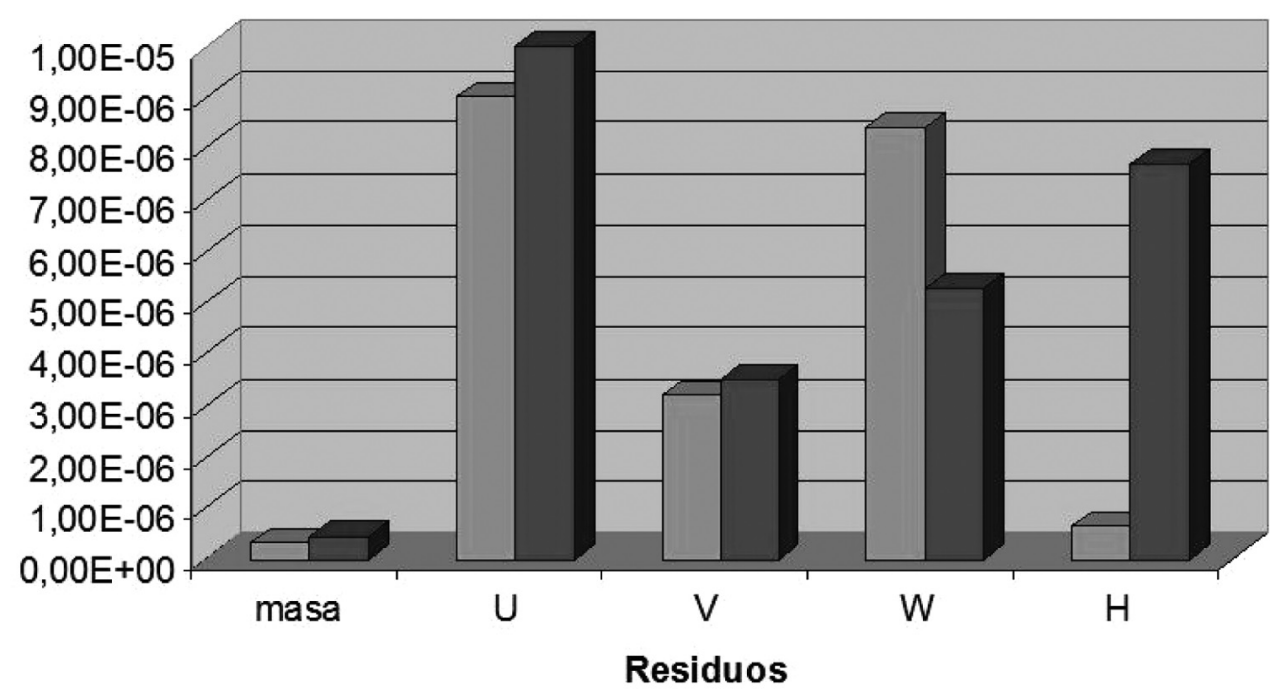

$\square$ Malla No Estructurada $\square$ Malla Estructurada

Figura 15. Comparación entre los residuos finales de cada variable. Fuente: (Rodríguez, 2007). 


\subsection{Comparación de los tiempos de cálculo de las simulaciones}

En la sección anterior se observó como el comportamiento de la convergencia varía para los dos casos simulados. Ahora es necesario realizar comparaciones entre los tiempos de cálculo de las simulaciones y los residuos finales de cada variable con el objeto de seleccionar adecuadamente el caso que mejor se adapte al problema simulado.

En la Figura 14 se muestra un gráfico de barras en el cual se comparan los tiempos de cálculo consumidos por los dos casos simulados.

Mediante la Figura 15 es posible hacer comparaciones entre los residuos finales obtenidos para las variables del flujo. En primer lugar se observa que para los dos casos simulados, la variable que presenta menores residuos es la Masa; esto indica que la ecuación de continuidad ha sido resuelta satisfactoriamente en los elementos del dominio y que la acumulación de masa en el sistema de distribución es pequeña. Por el contrario, la variable que presenta mayores residuos finales es la Velocidad U para ambos casos; este resultado parece lógico partiendo del hecho de que la Velocidad U es la componente de la velocidad que se encuentra en la dirección del flujo y por ende, es la que sufre mayores fluctuaciones a lo largo del dominio.

Para la variable Entalpía se observa que el caso de mallado estructurado presenta mayores residuos finales que el caso de mallado no estructurado; esto indica que este último tipo de discretización permite una resolución más eficiente de la ecuación de conservación de la energía en los volúmenes de control del dominio y por ende, realiza un mejor modelado del fenómeno de transferencia de calor en el problema simulado.

Cuadro 1. Dimensiones de los paneles de poliuretano

$\begin{array}{lr}\text { Espesor láminas de aluminio } & 8 \cdot 10^{-5} \mathrm{~m} \\ \text { Espesor poliuretano } & 2 \cdot 10^{-2} \mathrm{~m} \\ \text { Espesor total del ducto } & 0,02008 \mathrm{~m}\end{array}$

Fuente: (Los autores)
Cuadro 2. Propiedades físicas y térmicas del poliuretano con recubrimiento de aluminio

$\begin{array}{lr}\text { Densidad }\left(\mathrm{kg} / \mathrm{m}^{3}\right) & \text { de } 48 \text { a } 50 \\ \text { Presión interna máxima }(\mathrm{Pa}) & 500 \\ \text { Rugosidad absoluta }(\mathrm{m}) & 0,00012 \\ \text { Conductividad térmica a } 283 \mathrm{~K}(\mathrm{~W} / \mathrm{m} \cdot \mathrm{K}) & 0,022 \\ \quad \text { Fuente: (Los autores) }\end{array}$

\section{CONCLUSIONES}

Los resultados obtenidos en este trabajo de investigación permitieron establecer las siguientes conclusiones:

1. El modelo matemático empleado en la investigación, en conjunto con la aplicación del modelo de turbulencia $\mathrm{k}-\varepsilon$, permitió predecir satisfactoriamente el comportamiento termofluidodinámico del aire en el sistema de distribución de aire acondicionado en estudio.

2. Los dos métodos de discretización espacial empleados: malla no estructurada y malla estructurada resultan eficientes, desde el punto de vista numérico, en la simulación del comportamiento del aire en sistemas de distribución de aire acondicionado.

3. Debido a los bajos residuos obtenidos para la masa como variable de flujo, es posible afirmar que en este problema la ecuación de continuidad ha sido resuelta satisfactoriamente en los elementos del dominio y que la acumulación de masa en el sistema es despreciable.

4. En el caso de mallado no estructurado se obtuvieron menores residuos para las variables de flujo masa, velocidad $\mathrm{U}$ y velocidad $\mathrm{V}$, que en el caso de mallado no estructurado.

5. Por ser la componente de la velocidad U la que sufre mayores fluctuaciones a lo largo del dominio, sus residuos resultan mayores a los de las demás variables del sistema.

6. El uso de un mallado no estructurado como método de discretización espacial en este tipo de sistemas, permite una resolución más eficiente de la ecuación de conservación de la energía en los volúmenes de control del dominio y por ende, realiza un mejor modelado del fenómeno de transferencia de calor. 


\section{RFERENCIAS BIBLIOGRÁFICAS}

ANSYS. (2002). CFX 5.7 User's Manual, Waterloo, Ontario, Canada.

Cho, N. \& Kim, N. (1998). Numerical investigation of fluid flow in an automotive HVAC Module. Korea: Institute for Advanced Engineering.

Emvin, P. (2000). The full multigrid method applied to turbulent flow in ventilated enclosures using structured and unstructured grids. Sweden: Chalmers University of Technology.

Janka, A. (2001). Analysis of an unstructured finite volume method. Francia: Institut National de Recherche en Informatique et en Automatique.

Peng, S. (1998). Modeling of turbulent flow and heat transferforbuilding ventilation. Sweden: Chalmers University of Technology.

Pérez, J. (2004). Simulación tridimensional del comportamiento fluidodinámico de una bomba hidráulica de discos. Tesis de grado para optar al título de Ingeniero Mecánico, Escuela de Ingeniería y Ciencias Aplicadas, Departamento de Mecánica, Universidad de Oriente, Barcelona, Venezuela.

Schneider, F. \& Maliska, C. (2002, Octubre). Solução numérica simultânea de escoamentos bidimensionais imcompressivveis pelo método dos volumes finitos usando malhas nãoestruturadas. Ponencia presentada en el IX Congresso Brasileiro de Engenharia e Ciências Térmicas, Brasil.

Rodríguez, L. \& Verde, A. (2005). Análisis numérico del comportamiento térmico y fluidodinámico del aire en un sistema de distribución de aire acondicionado, Tesis de grado para optar al título de Ingeniero
Mecánico, Escuela de Ingeniería y Ciencias Aplicadas, Departamento de Mecánica Universidad de Oriente, Barcelona, Venezuela.

\section{SOBRE LOS AUTORES}

\section{Luz Rodríguez Collado}

Centro de Termofluidodinámica y Mantenimiento (CTYM), Universidad de Oriente.

Puerto La Cruz, Anzoátegui, Venezuela

Teléfono: 584166814777

Facsímil: 582814203270

Correo electrónico: luzmarc2003@gmail.com

\section{María Collado Contreras}

Centro de Termofluidodinámica y Mantenimiento (CTYM), Universidad de Oriente.

Puerto La Cruz, Anzoátegui, Venezuela

Teléfono: 584166822121

Facsímil: 582814203270

Correo electrónico: mariacolladoc@ cantv.net

\section{Edgar Rodríguez Malaver}

Centro de Termofluidodinámica y Mantenimiento (CTYM), Universidad de Oriente.

Puerto La Cruz, Anzoátegui, Venezuela

Teléfono: 584166814132

Facsímil: 582814203270

Correo electrónico: rmedgar5@cantv.net

\section{Henry Espinoza Bejarano}

Centro de Termofluidodinámica y Mantenimiento (CTYM), Universidad de Oriente.

Puerto La Cruz, Anzoátegui, Venezuela

Teléfono: 584166810915

Facsímil: 582814203270

Correo electrónico: hespinoza@cantv.net 